\title{
Evaluation of soluble mesothelin-related peptides and MSLN genetic variability in asbestos-related diseases
}

\author{
Katja Goricar ${ }^{1}$, Viljem Kovac ${ }^{2,3}$, Metoda Dodic-Fikfak³,4, Vita Dolzan¹, Alenka Franko ${ }^{3,4}$ \\ ${ }_{1}^{1}$ Pharmacogenetics Laboratory, Institute of Biochemistry, Faculty of Medicine, University of Ljubljana, Ljubljana, Slovenia \\ 2 Institute of Oncology Ljubljana, Ljubljana, Slovenia \\ ${ }^{3}$ Faculty of Medicine, University of Ljubljana, Ljubljana, Slovenia \\ ${ }^{4}$ Clinical Institute of Occupational Medicine, University Medical Centre Ljubljana, Ljubljana, Slovenia
}

Radiol Oncol 2020; 54(1): 86-95.

Received 10 January 2020

Accepted 27 January 2020

Disclosure: No potential conflicts of interest were disclosed.

Correspondence to: Assoc. Prof Alenka Franko, M.D., Ph.D., Clinical Institute of Occupational Medicine, University Medical Center Ljubljana, Ljubljana, Poljanski nasip 58, SI-1000 Ljubljana, Slovenia. Phone: +38615222119; Fax: +386 1522 2478; E-mail: alenka.franko@siol.net

Background. Asbestos exposure is associated with increased risk of several diseases, including malignant mesothelioma (MM). Cell surface glycoprotein mesothelin is overexpressed in MM and serum soluble mesothelin-related peptides (SMRP) were already proposed as a diagnostic or prognostic biomarker in MM. However, interindividual variability in serum SMRP levels limits the clinical usefulness. Our primary objective was to investigate the influence of MSLN rs 1057147 on serum SMRP levels in asbestos-exposed subjects and patients with asbestos-related diseases as well as on survival in MM.

Subjects and methods. Among 782 asbestos-exposed subjects and patients with asbestos-related diseases, 154 had MM. Serum SMRP levels were determined using sandwich enzyme-linked immunosorbent assay. All subjects were genotyped for MSLN rs 1057147 polymorphism using competitive allele-specific polymerase chain reaction. Nonparametric tests, logistic and Cox regression were used in statistical analysis to compare different subject groups. Results. MM patients had significantly higher SMRP levels than all other subjects $(p<0.001)$. Compared to wild-type MSLN rs 1057147 genotype, both heterozygotes and carriers of two polymorphic alleles had significantly higher SMRP levels among subjects without MM ( $p<0.001)$, but not in MM patients $(p=0.424)$. If genotype information was included, specificity of SMRP increased from $88.5 \%$ to $92.7 \%$ for the optimal cutoff value. Overall survival was significantly shorter in MM patients carrying at least one polymorphic rs 1057147 allele ( $\mathrm{HR}=1.72,95 \% \mathrm{Cl}=1.15-2.55, p=0.008)$.

Conclusions. MSLN genetic variability affects serum SMRP levels and was associated with shorter survival of MM patients. Combination of genetic and serum factors could therefore serve as a better diagnostic or prognostic biomarker in MM patients.

Key words: malignant mesothelioma; asbestos-related disease; mesothelin, soluble mesothelin-related peptides; polymorphism

\section{Introduction}

Occupational and environmental exposure to asbestos is associated with the development of different asbestos-related diseases. Even though several countries have banned the use of asbestos after it was classified as a carcinogen in 1977, it is still being used in some countries and it is also still present in the environment. ${ }^{1,2}$ Additionally, there is a long latency period between exposure and development of asbestos-related diseases, which can occur several decades after asbestos exposure even in subjects exposed to relatively low doses. ${ }^{1}$ Therefore, the incidence of asbestos-related diseases continues to rise in most countries and they remain one of the major public health issues. ${ }^{1}$ 
Pleural plaques, diffuse pleural thickening, pleural effusions and asbestosis are classified as benign asbestos-related diseases. ${ }^{1}$ Exposure to asbestos also increases the risk of various cancers, including malignant mesothelioma (MM). $\mathrm{MM}$ is an extremely aggressive cancer affecting serosal membranes, mostly pleura or peritoneum. ${ }^{3,4}$ Due to non-specific symptoms, diagnosis is usually made in the advanced stages of the disease, leading to poor prognosis and short survival of MM patients. Even though the use of chemotherapy increased the survival of MM patients, response rate is still limited. ${ }^{4-6}$ Early diagnosis could therefore contribute to a more effective treatment of MM. ${ }^{7,8}$ Currently, immunohistochemical analysis investigating a panel of markers on tissue samples is required to confirm the MM diagnosis. ${ }^{9}$ New noninvasive biomarkers that would enable earlier diagnosis of MM are thus extensively studied, particularly in pleural MM.

The most frequently investigated biomarker in $\mathrm{MM}$ is mesothelin, as many studies have shown it is frequently increased in both tumor tissue and serum of MM patients, especially in epithelioid histological type. ${ }^{8,10-17}$ Mesothelin is a cell-surface glycoprotein expressed in mesothelial cells and overexpressed in several cancer types. It is involved in important cellular processes, including cell adhesion, proliferation, invasion, and epithelial-to-mesenchymal transition. ${ }^{18,19}$ Mesothelin is a glycophosphatidylinositol-linked membrane protein, but it also has three isoforms that are present in the circulation. 12,15,20 Enzyme-linked immunosorbent assay (ELISA) can detect different isoforms, usually referred to as soluble mesothelin related peptides (SMRP). ${ }^{12,20}$

Meta-analyses focusing on SMRP as a diagnostic marker of MM showed that high SMRP has high specificity, but limited sensitivity, suggesting that positive results should lead to further diagnostic steps, but negative results do not exclude MM and therefore additional biomarkers are needed..$^{12,14}$

Several studies also investigated SMRP as a prognostic biomarker in MM. ${ }^{18,21-23}$ SMRP levels were markedly increased at disease progression. ${ }^{21}$ In a meta-analysis, increased SMRP was associated with shorter overall survival and worse prognosis. ${ }^{18}$ Numerous studies also noted that serial longitudinal SMRP measurements may be more informative than SMRP levels at diagnosis. ${ }^{22,23}$ Mesomark $^{\mathrm{TM}}$ ELISA kit was already approved by the FDA for the measurement of SMRP and monitoring of MM. ${ }^{20,24}$

A few previous studies have shown that genetic variability also influences SMRP levels. Single nucleotide polymorphisms (SNPs) in the $5^{\prime}$ and $3^{\prime}$ untranslated region (UTR) of the mesothelin gene (MSLN) can contribute to the observed SMRP variability. Polymorphic alleles of rs3764247 (c.$894 \mathrm{~A}>\mathrm{C}$ ), rs3764246 (c. $-790 \mathrm{~A}>\mathrm{G}$ ), and rs2235503 (c.-340C $>A$ ) in the 5 ' UTR were associated with increased SMRP levels in healthy asbestos-exposed subjects or subjects with benign asbestos-related diseases, but not in MM patients. ${ }^{25,26}$ Similarly, rs1057147 (c. ${ }^{*} 69 \mathrm{G}>\mathrm{A}$ ) in the $3^{\prime}$ UTR that affects miR-611 binding was also associated with increased SMRP levels in healthy asbestos-exposed subjects or subjects with benign asbestos-related diseases. ${ }^{27,28}$ MSLN genetic variability was thus shown to improve specificity of SMRP as a diagnostic marker and could help redefine and improve the predictive ability of currently used cutoff values. ${ }^{25,26,28}$ However, no data is available regarding the association of MSLN genetic variability with the prognosis of MM.

The aim of our study was to determine serum SMRP levels in patients with asbestos-related diseases and in asbestos-exposed subjects without asbestos-related disease and to assess the association of MSLN rs1057147 with serum SMRP levels. We also investigated the association of serum SMRP levels and MSLN rs1057147 with the survival of MM patients.

\section{Subjects and methods}

\section{Subjects}

The study included 782 subjects with different asbestos-related diseases (pleural plaques, asbestosis and $\mathrm{MM}$ ) and asbestos-exposed subjects with no asbestos-related disease.

Patients with MM were treated at the Institute of Oncology Ljubljana in the period between 1 January 2004 and 31 December 2012. The diagnosis of pleural MM was performed by thoracoscopy and the diagnosis of peritoneal MM by laparoscopy. In both cases, the diagnosis of MM was confirmed histologically by an experienced pathologist. The MM stage was determined according to the TNM staging system for pleural MM, while performance status was evaluated according to Eastern Cooperative Oncology Group (ECOG) scores.

All patients with pleural plaques, asbestosis and subjects with no asbestos-related disease were occupationally exposed to asbestos and presented at the State Board for the Recognition of Occupational Asbestos Diseases in the period from 1 January 1998 to 31 December 2007. The diagnosis of pleural 
plaques, asbestosis or "no asbestos-related disease" was based on the Helsinki Criteria for Diagnosis and Attribution of Asbestos Diseases ${ }^{29}$ and on the American Thoracic Society recommendations ${ }^{30}$ and was confirmed by two groups of experts each consisting of a skilled occupational physician, a radiologist, and a pulmonologist. Follow-up was performed in all patients in 2018 to confirm they did not develop any other asbestos-related disease.

Demographic and clinical data were obtained from the medical records. Data on smoking were obtained using a standardized questionnaire ${ }^{31,32}$ and during the interview. Patients were classified as ever/never smokers. All subjects provided written informed consent. The study was approved by the National Medical Ethics Committee of the Republic of Slovenia and was carried out according to the Helsinki Declaration.

\section{Serum SMRP measurement}

Serum samples were collected at diagnosis for patients with $\mathrm{MM}$ and at inclusion in the study for all other subjects. Serum samples were prepared within 6 hours after blood sampling, aliquoted and stored at $-20^{\circ} \mathrm{C}$. For determining serum SMRP levels, sandwich ELISA assay (Mesomark ${ }^{\mathrm{TM}}$ ) using two monoclonal antibodies (4H3 and OV569) was used according to the manufacturer's protocol (Fujirebio Europe BV, Breda, The Netherlands). ${ }^{24}$

\section{DNA extraction and genotyping}

Genomic DNA was extracted from peripheral blood leukocytes using Qiagen FlexiGene Kit (Qiagen, Hilden, Germany). MSLN rs1057147 (c.*69G>A) genotype was determined for all subjects using a fluorescent-based competitive allele-specific polymerase chain reaction (KASPar) assay (LGC Genomics, UK) or real-time PCR-based Taqman assay (Applied Biosystems, Foster City, CA, USA) following the manufacturer's instructions. In 15\% of the subjects, samples were genotyped in duplicates and the duplicate call rate was $100 \%$.

\section{Statistical analysis}

Continuous variables were described using median with interquartile range $(25 \%-75 \%)$ or $95 \%$ confidence intervals (CIs), while categorical variables were described using frequencies. Fisher's exact test was used to compare categorical variables among different groups. Nonparametric Mann-Whitney or Kruskal-Wallis tests were used to compare distribution of continuous variables. Pairwise comparisons with post hoc Bonferroni corrections were used with Kruskal-Wallis test to obtain adjusted $p$ values $\left(p_{\text {adj }}\right)$. Deviation from the Hardy-Weinberg equilibrium (HWE) was evaluated using the standard chi-square test. Both dominant and additive genetic models were used in the analysis. Logistic regression models were used to calculate non-adjusted and adjusted odds ratios (ORs) and 95\% CIs for comparison of genotype frequencies between groups. Clinical characteristics, significant in univariable analysis, were used for further adjustment. A receiver operating characteristic (ROC) curve was used to analyze the predictive value and the area under the curve (AUC).

In the survival analysis, progression-free survival (PFS) and overall survival (OS) were assessed. PFS was defined as the time to the day of documented disease progression, or death, whichever occured first and OS was defined as the time to death from any cause. Patients without progression or death at the time of the analysis were censored at the date of the last follow-up. The data on vital status were obtained from medical records or from the Slovenian Cancer Registry. Kaplan-Meier analysis was used to calculate median survival or follow-up time, while Cox regression was used to calculate the hazard ratios (HR) with the 95\% CIs.

The statistical analyses were carried out by using IBM SPSS Statistics version 21.0 (IBM Corporation, Armonk, NY, USA). All statistical tests were twosided and the level of significance was set at 0.05 .

\section{Results}

Among 782 subjects included in our study, 154 (19.7\%) patients had MM. Among 628 non-MM subjects that were occupationally exposed to asbestos, $69 \mathrm{did}$ not develop any asbestos-related disease, 410 subjects had pleural plaques, and 149 patients had asbestosis. Characteristics of each subject group are presented in Table 1. Gender and smoking distributions were similar across all groups ( $p$ $=0.479$ and 0.740 , respectively). On the other hand, age at diagnosis or inclusion in the study differed significantly between subject groups (KruskalWallis test statistic $=88.602 ; p<0.001)$. MM patients were significantly older compared to all other subjects (Mann-Whitney $\mathrm{U}=69560.5 ; p<0.001$ ).

Among 138 patients with pleural MM, 8 (5.8\%) had stage 1, $41(29.7 \%)$ stage 2, 43 (31.2\%) stage 3 , and $41(29.7 \%)$ stage 4 disease, while the stage could not be determined in $5(3.6 \%)$ patients. 
TABLE 1. Clinical characteristics of study groups

\begin{tabular}{|c|c|c|c|c|c|c|c|}
\hline Characteristic & & $\begin{array}{l}\text { All subjects } \\
(\mathrm{N}=782)\end{array}$ & $\begin{array}{l}\text { No disease } \\
(\mathrm{N}=69)\end{array}$ & $\begin{array}{l}\text { Pleural plaques } \\
\qquad(\mathrm{N}=410)\end{array}$ & $\begin{array}{l}\text { Asbestosis } \\
(\mathrm{N}=149)\end{array}$ & $\begin{array}{c}M M \\
(N=154)\end{array}$ & $\begin{array}{l}\text { MM with SMRP } \\
\text { at diagnosis } \\
(\mathrm{N}=86)\end{array}$ \\
\hline Gender & Male, N (\%) & $581(74.3)$ & $52(75.4)$ & $295(72.0)$ & $115(77.2)$ & 119 (77.3) & 69 (80.2) \\
\hline Age & $\begin{array}{l}\text { Years, median } \\
(25 \%-75 \%)\end{array}$ & $56.9(50.2-64.9)$ & $52.9(48.2-59.2)$ & $54.6(48.8-62.2)$ & $59.1(51.2-65.2)$ & 65 (57-70) & $66(59-72)$ \\
\hline Smoking & No, N (\%) & $375(49.0)$ [17] & $31(45.6)[1]$ & $203(50.9)$ [1 ] & $71(48.0)[1]$ & $70(46.7)[4]$ & 43 (51.2) [2] \\
\hline SMRP & $\begin{array}{c}\text { nmol/l, } \\
\text { median } \\
(25 \%-75 \%)\end{array}$ & & $\begin{array}{c}0.30 \\
(0.00-0.85)[1]\end{array}$ & $\begin{array}{c}0.14 \\
(0.00-0.58)[6]\end{array}$ & $\begin{array}{c}0.03 \\
(0.00-0.39)[5]\end{array}$ & & $2.43(0.44-8.62)$ \\
\hline
\end{tabular}

Number of missing data is presented in [] brackets.

MM = malignant mesothelioma; SMRP = soluble mesothelin-related peptides

Additionally, 16 (10.4\%) patients had peritoneal MM. Most patients had epithelioid MM (114, $74.0 \%), 15(9.7 \%)$ patients had biphasic and 14 (9.1\%) patients had sarcomatoid MM. In the remaining $11(7.1 \%)$ patients histological type could not be determined. ECOG performance status at diagnosis was 0 in $4(2.6 \%)$ patients, 1 in $82(53.2 \%)$, 2 in $60(39.2 \%)$ and 3 in $3(1.9 \%)$ MM patients, while no data on performance status were available for 5 (3.2\%) patients.

Serum samples of 86 (55.8\%) MM patients were available at diagnosis, therefore the SMRP level was measured only in these patients. MM patients with available data on serum SMRP at diagnosis did not differ significantly from the rest of MM patients regarding gender, smoking, stage, location (pleura or peritoneum) and histological type (all $p>0.05)$. However, these patients were older ( $p=$ $0.005)$ and had worse ECOG performance status ( $p$ $=0.019$ ).

\section{Serum SMRP levels}

SMRP serum levels differed significantly between subject groups presented in Table 1 (KruskalWallis test statistic $=96.470 ; p<0.001$; Figure 1A). MM patients had significantly higher SMRP levels at diagnosis after Bonferroni correction for multiple comparisons than subjects with no disease (Kruskal-Wallis test statistic $=-158.068 ; p_{\text {adj }}$ $<0.001$ ), subjects with pleural plaques (KruskalWallis test statistic $\left.=-209.046 ; p_{\text {adj }}<0.001\right)$ and patients with asbestosis (Kruskal-Wallis test statistic $\left.=-246.125 ; p_{\text {adj }}<0.001\right)$. Additionally, the difference between patients with asbestosis and subjects with no disease remained significant after Bonferroni correction (Kruskal-Wallis test statistic 88.058; $\left.p_{\text {adj }}=0.015\right)$, while no significant differences after adjustment were observed between subjects with pleural plaques and subjects with no disease (Kruskal-Wallis test statistic $\left.=50.979 ; p_{\text {adj }}=0.294\right)$ or patients with asbestosis (Kruskal-Wallis test statistic $\left.=37.079 ; p_{\text {adj }}=0.318\right)$.

When comparing MM patients with all other subjects, MM patients had significantly higher SMRP levels (2.43 (0.44-8.62) nmol/l compared to 0.13 (0.00-0.55) nmol/1; Mann-Whitney U = 42493; $p<0.001)$. In the ROC curve analysis, the AUC for serum SMRP predicting MM was $0.802(95 \% \mathrm{CI}$ $=0.740-0.864 ; p<0.001$, Figure 1B). At the cutoff
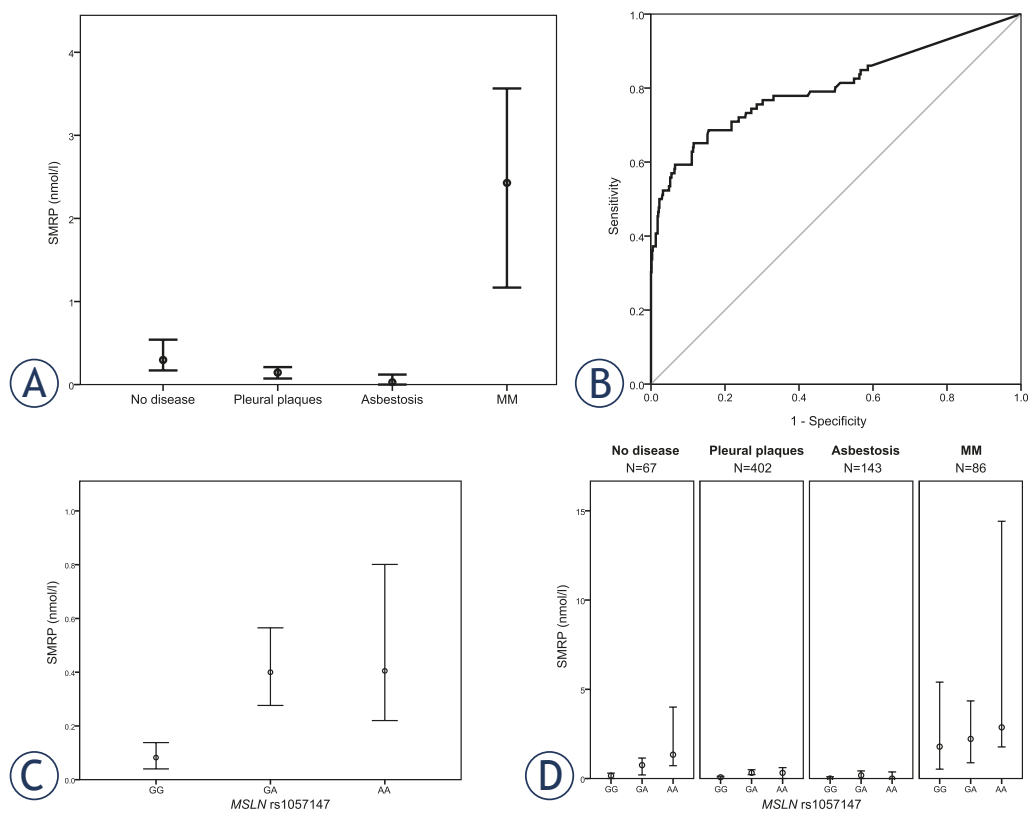

FIGURE 1. Median soluble mesothelin-related peptides (SMRP) levels in different groups (A), receiver operating characteristic (ROC) curve for SMRP predicting malignant mesothelioma (MM) (B) and association of MSLN rs 1057147 genotype with serum SMRP levels at inclusion in the study in the whole cohort of 698 subjects (C) and for each subject group (D). Data are presented as median with $95 \%$ confidence intervals. 
TABLE 2. ROC curve analysis according to MSLN rs 1057147 genotype

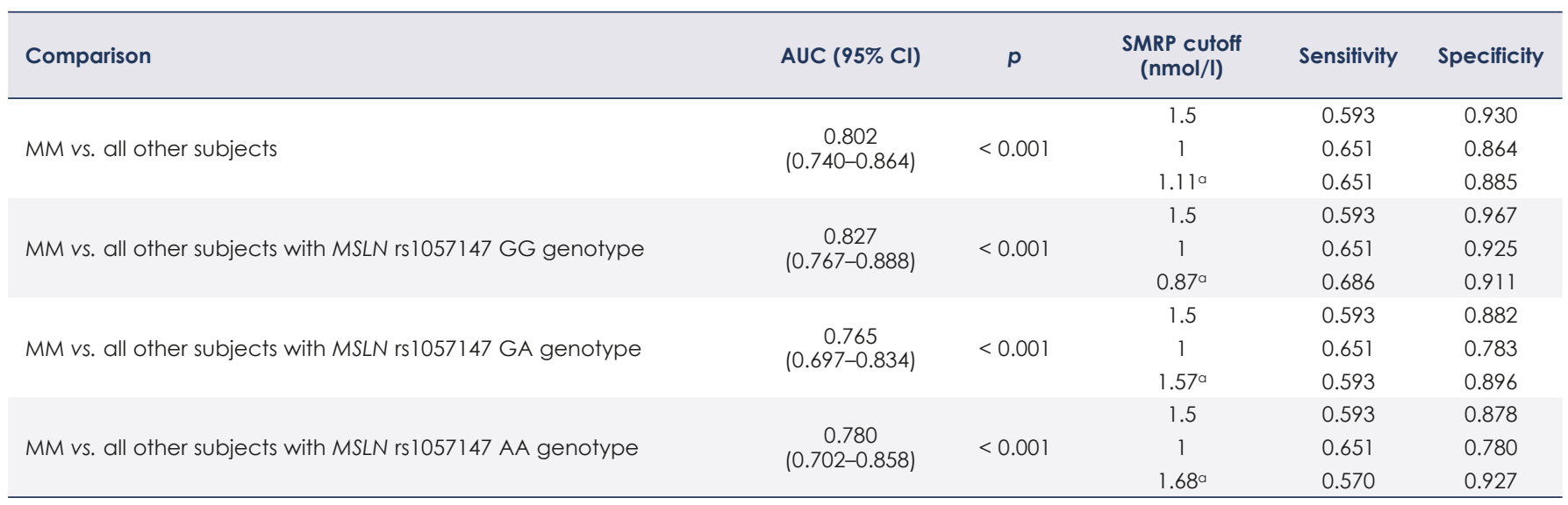

a Cutoff with the highest sum of sensitivity and specificity.

$\mathrm{AUC}=$ area under the curve; $\mathrm{MM}=$ malignant mesothelioma; $\mathrm{ROC}=$ receiver operating characteristic; $\mathrm{SMRP}=$ soluble mesothelin-related peptides

TABLE 3. Odds for MM in subjects with different MSLN rs 1057147 genotypes

\begin{tabular}{|c|c|c|c|c|c|c|c|c|}
\hline \multirow{2}{*}{ Genotype } & \multirow{2}{*}{$\begin{array}{c}\text { No disease } \\
(\mathrm{N}=69) \\
\mathrm{N}(\%)\end{array}$} & \multirow{2}{*}{$\begin{array}{c}\text { Pleural plaques } \\
(\mathrm{N}=410) \\
\mathrm{N}(\%)\end{array}$} & \multirow{2}{*}{$\begin{array}{c}\text { Asbestosis } \\
(\mathrm{N}=149) \\
\mathrm{N}(\%)\end{array}$} & \multirow{2}{*}{$\begin{array}{c}M M \\
(N=154) \\
N(\%)\end{array}$} & \multicolumn{4}{|c|}{ MM vs all other subjects } \\
\hline & & & & & OR $(95 \% \mathrm{Cl})$ & $p$ & $\mathrm{OR}_{\mathrm{adj}}(95 \% \mathrm{Cl})$ & $p_{\text {adj }}$ \\
\hline GG & $41(60.3)[1]$ & 241 (59.1) [2] & $84(56.8)[1]$ & $81(52.6)$ & reference & & reference & \\
\hline AA & $6(8.8)$ & $20(4.9)$ & $15(10.1)$ & $13(8.4)$ & $1.43(0.73-2.80)$ & 0.292 & $1.30(0.64-2.64)$ & 0.472 \\
\hline$G A+A A$ & $27(39.7)$ & $167(40.9)$ & $64(43.2)$ & $73(47.4)$ & $1.28(0.90-1.82)$ & 0.174 & $1.29(0.89-1.87)$ & 0.187 \\
\hline
\end{tabular}

value of $1.5 \mathrm{nmol} / 1$ suggested by the ELISA assay manufacturer, specificity for predicting $\mathrm{MM}$ was 0.930 , while sensitivity was 0.593 . However, we observed the highest sum of specificity and sensitivity at the cutoff value of $1.11 \mathrm{nmol} / \mathrm{l}$, resulting in a specificity of 0.885 and sensitivity of 0.651 (Table 2).

Serum SMRP was not associated with gender (Mann-Whitney $\mathrm{U}=48033.5 ; p=0.756$ ) or smoking (Mann-Whitney $\mathrm{U}=61928.5 ; p=0.276$ ). In the whole study group, higher age was weakly correlated with slightly higher SMRP (Spearman's rho = $0.105 ; p=0.005)$, but within each group, there was no association between age and SMRP (no disease: $p=0.127$; pleural plaques: $p=0.856$; asbestosis: $p=$ 0.846; MM: $p=0.866$ ).

In MM patients, 10 patients with peritoneal MM had significantly higher SMRP levels than patients with pleural MM (8.72 (3.65-12.25) nmol/1 vs. 1.78 (0.267.74) nmol/l, Mann-Whitney $\mathrm{U}=569$; $p=0.011$ ). Additionally, patients with epithelioid histological type had significantly higher SMRP levels than patients with other histological types
(2.87 (0.51-10.47) nmol/l vs. 1.14 (0.10-3.60) nmol/1; Mann-Whitney $\mathrm{U}=540.5 ; p=0.035$ ).

\section{MSLN rs1057147 genotype frequencies}

Genotype frequencies of MSLN rs1057147 are presented in Table 3. Minor allele frequency was $24.6 \%$ in the whole cohort. Genotype frequencies were in agreement with HWE in subjects without any disease as well as in the whole cohort (all $p>$ 0.05). MSLN rs1057147 was not associated with the odds for developing MM and there were no differences between non-adjusted and age-adjusted ORs (Table 3). Similarly, MSLN rs1057147 was not associated with the odds for developing any asbestos-related disease, individually or combined, compared to subjects with no disease (all $p>0.05$, unadjusted ORs in Supplementary Table S1).

\section{MSLN rs1057147 and serum SMRP levels}

SMRP levels differed significantly between MSLN rs1057147 genotype groups in all 698 subjects with 
TABLE 4. Serum SMRP levels according to MSLN rs 1057147 genotype

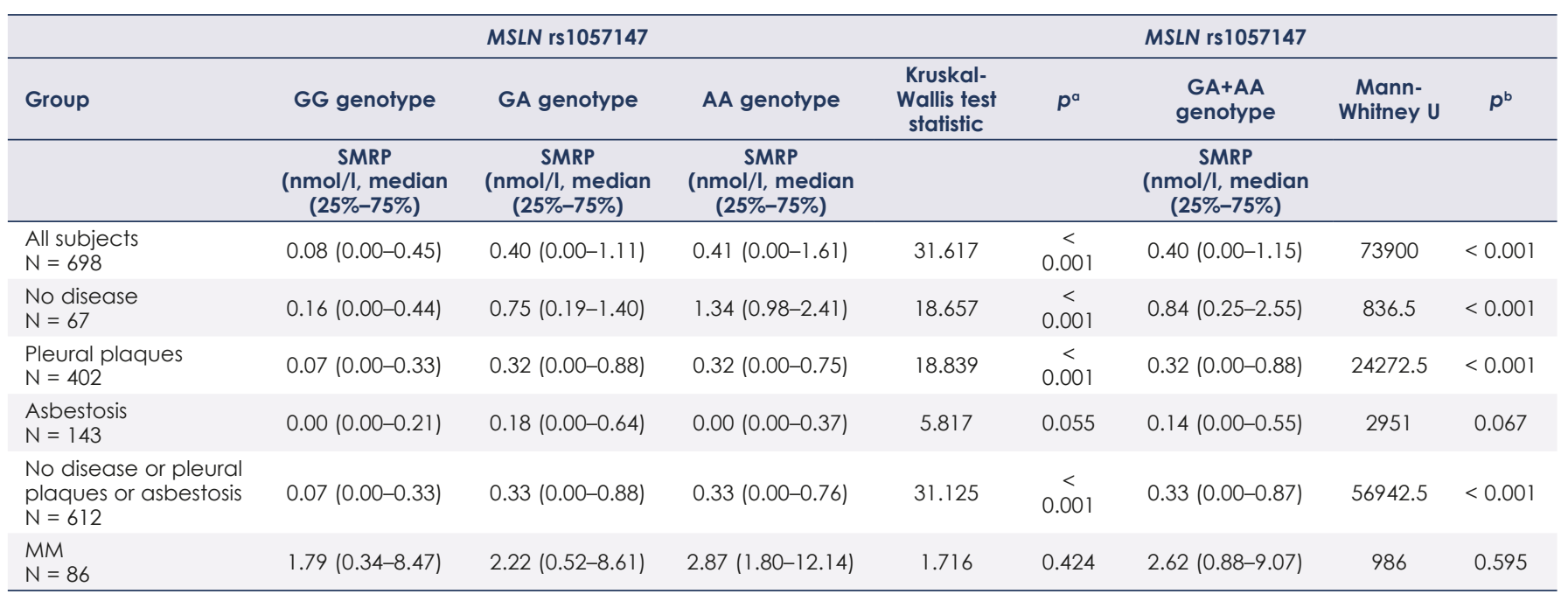

additive model; ${ }^{b}$ dominant model

MM = malignant mesothelioma; SMRP = soluble mesothelin-related peptides

available SMRP levels and MSLN genotype $(p<$ 0.001; Table 4; Figure 1C). Compared to carriers of two wild-type alleles, both heterozygotes and carriers of two polymorphic alleles had significantly higher SMRP levels after Bonferroni correction (Kruskal-Wallis test statistic $=-83.315, p_{\text {adj }}<0.001$ and Kruskal-Wallis test statistic $=-90.291 ; p_{\mathrm{adj}}=$ 0.007 , respectively). The difference was also significant in the dominant model $(p<0.001)$. When we analyzed the role of MSLN rs1057147 in each group separately (Table 4, Figure 1D), SMRP levels differed significantly between genotype groups in subjects without any disease, all subjects without MM or subjects with pleural plaques in both additive and dominant model (all $p<0.001$ ). However, in patients with asbestosis or MM, MSLN rs1057147 was not significantly associated with SMRP levels at diagnosis in additive $(p=0.055$ and $p=0.424$, respectively) or dominant model ( $p=0.067$ and $p=$ 0.595 , respectively), even though carriers of polymorphic allele had higher SMRP levels.

As genetic variability only influenced the SMRP levels in non-MM subjects, we performed separate ROC curve analyses stratified by genotype, comparing all MM patients to non-MM subjects with individual genotypes. The predictive capacity of SMRP was genotype-dependent. The AUC was the highest when comparing the MM patients to all other subjects with MSLN rs1057147 GG genotype and SMRP had the highest specificity (Table 2). Similarly, the cutoff value with the highest sum of specificity and sensitivity differed according to genotype. The cutoff was $0.87 \mathrm{nmol} / 1$ in subjects with GG genotype, but $1.68 \mathrm{nmol} / \mathrm{l}$ in subjects with AA genotype (Table 2).

\section{MSLN rs1057147 and survival of MM patients}

Median PFS of MM patients was 7.8 (5.5-13.6) months, while median OS was 18.0 (9.8-29.6) months. The follow-up time of censored patients was 43.5 (21.8-94.3) months.

Compared to MSLN rs1057147 GG genotype, patients with GA and AA genotypes had non-significantly shorter PFS, which is supported by the slightly elevated HRs $(\mathrm{HR}=1.39,95 \% \mathrm{CI}=0.96-$ 2.01; $p=0.084$ and $\mathrm{HR}=1.40,95 \% \mathrm{CI}=0.74-2.66$;
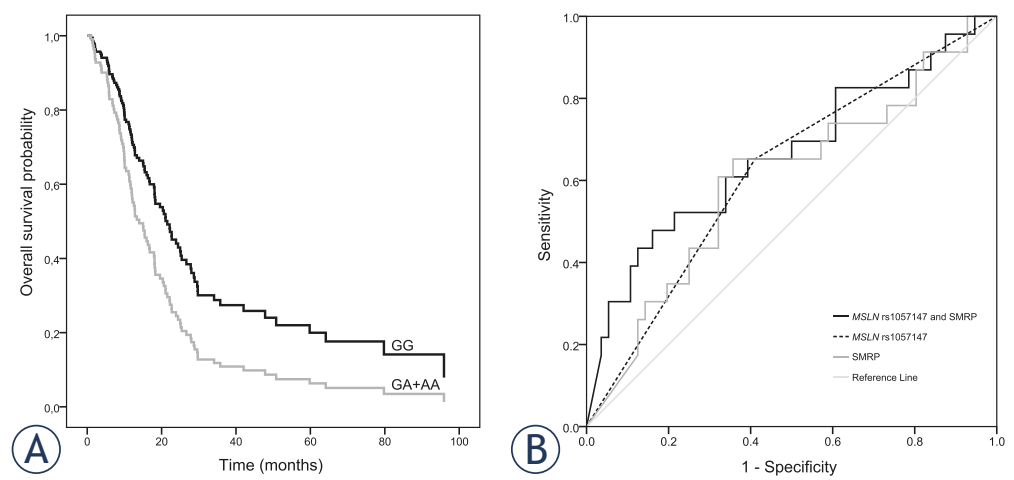

FIGURE 2. The influence of MSLN rs1057147 genotype on overall survival (A) and receiver operating characteristic (ROC) curve for MSLN rs 1057147 and soluble mesothelin-related peptides (SMRP) predicting overall survival above median (B). 
$p=0.306$ for GA and AA genotypes, respectively). Similar results were observed in the dominant model $(\mathrm{HR}=1.39,95 \% \mathrm{CI}=0.98-1.98 ; p=0.068)$ (Supplementary Table S2).

Compared to MSLN rs1057147 GG genotype, patients with GA and AA genotypes had shorter OS (Supplementary Table S2). The difference was statistically significant for GA genotype $(\mathrm{HR}=$ $1.68,95 \% \mathrm{CI}=1.11-2.54 ; p=0.015)$, while only a trend was seen in AA genotype ( $\mathrm{HR}=1.91,95 \%$ $\mathrm{CI}=0.96-3.81 ; p=0.066)$. In the dominant model, median OS was significantly shorter in carriers of at least one polymorphic allele compared to carriers of two wild-type alleles (15.1 months vs. 22.2 months, $\mathrm{HR}=1.72,95 \% \mathrm{CI}=1.15-2.55 ; p=0.008$; Figure 2A).

In MM patients whose SMRP levels were available at diagnosis, the serum SMRP level was not significantly associated with PFS or OS in our study group $(\mathrm{HR}=0.99,95 \% \mathrm{CI}=0.96-1.02 ; p=0.574$ and $\mathrm{HR}=1.03,95 \% \mathrm{CI}=0.99-1.06 ; p=0.110$, respectively). Still, patients with SMRP above $1.5 \mathrm{nmol} / 1$ had slightly though not significantly shorter OS (12.1 months vs. 20.6 months, $\mathrm{HR}=1.45,95 \% \mathrm{CI}=$ $0.80-2.61 ; p=0.220)$. In a model combining the serum SMRP level at diagnosis and MSLN rs1057147, MSLN rs1057147 was the most important predictor of OS (HR $=2.65,95 \% \mathrm{CI}=1.41-4.99 ; p=0.002)$.

Using the ROC curve analysis, we assessed how well MSLN rs1057147 can predict OS above the median of 18 months in MM patients whose SMRP levels were available at diagnosis (Figure 2B). For MSLN rs1057147 in the dominant model, the AUC was $0.621(0.485-0.757)$ and did not reach statistical significance $(p=0.093)$. For the SMRP level at diagnosis, the AUC was $0.602(0.460-0.743)$ and did not reach statistical significance $(p=0.157)$. On the other hand, additive combination of both MSLN rs1057147 and the SMRP level at diagnosis based on the Cox regression model that included both variables increased the AUC to 0.662 (0.521-0.803) and was a significant predictor of OS above 18 months $(p=0.024)$.

\section{Discussion}

In the present study, we evaluated serum SMRP as a biomarker in the context of MSLN genetic variability in a large group of subjects with asbestosrelated diseases. We also evaluated the association of SMRP and MSLN genetic variability with the survival of MM patients. We showed that serum SMRP is increased in MM patients and that MSLN rs1057147 affects serum SMRP levels in subjects without MM, which could influence the selection of optimal cutoff values for MM diagnosis. Additionally, MSLN rs1057147 was an independent predictor of OS in MM patients.

Our study showed that serum SMRP is significantly increased in MM patients compared to patients with other asbestos-related diseases or asbestos-exposed subjects without any disease. All available meta-analyses confirmed that SMRP is elevated in the serum of MM patients as compared to healthy individuals, patients with asbestos-related diseases, benign respiratory diseases or lung cancer. ${ }^{12,14,16}$ Previous studies suggest that serum SMRP levels are the highest in epithelioid $\mathrm{MM}$, which is consistent with our results. ${ }^{12,14}$ Our present study also included patients with peritoneal $\mathrm{MM}$ who were rarely represented in other studies or meta-analyses that focused on pleural MM. Interestingly, we observed that patients with peritoneal MM had significantly higher SMRP than patients with pleural MM. Our results are in agreement with a recent study that also reported elevated serum SMRP in patients with peritoneal MM compared to controls. ${ }^{33}$ This suggests that studies on a larger numbers of patients with peritoneal MM are needed to elucidate if SMRP could also serve as a diagnostic biomarker in these patients.

Studies therefore suggest that SMRP measurement could be a potential screening tool for the detection of MM, but clinical use is still limited due to the probability of false positive and especially false negative results. ${ }^{12,14,16}$ Some issues related to sensitivity may be associated with a difficult selection of an appropriate cutoff value and different studies found different optimal thresholds. ${ }^{12,14,16}$ In our study, the standard cutoff of $1.5 \mathrm{nmol} / 1$ had $93 \%$ specificity and $59.3 \%$ sensitivity for distinguishing MM patients from other subjects. The cutoff with the best sum of specificity and sensitivity was 1.11 nmol/l, with $88.5 \%$ specificity and $65.1 \%$ sensitivity.

Genetic factors that affect gene expression may account for some of the observed interindividual variability in serum SMRP levels and affect the usefulness of SMRP as a biomarker. In the present study, polymorphic MSLN rs1057147 allele was associated with significantly higher serum SMRP levels in both additive and dominant models in the entire cohort. When analyzing each subject group separately, a significant association between MSLN rs1057147 and serum SMRP was seen in subjects without a disease and with pleural plaques, while only a trend was observed in asbestosis patients. 
Among MM patients, carriers of polymorphic allele had slightly higher serum SMRP, however, there was a large variability in serum SMRP levels and the differences among genotypes were not significant. Both previously published studies focusing on MSLN rs1057147 have shown that this polymorphism is associated with increased serum SMRP levels among subjects without MM..$^{27,28}$ Consistent with our results, this SNP was not associated with SMRP levels in MM patients. ${ }^{28}$ MSLN rs1057147 is located in the $3^{\prime}$ UTR region within a binding site for miR-611. ${ }^{28}$ Polymorphic allele could reduce the strength of binding to miRNA, limiting the inhibition of gene translation that could lead to the observed increased SMRP levels. ${ }^{27}$

However, other mechanisms might be more important for the increased SMRP expression seen in MM..$^{28}$ MSLN rs1057147 as a standalone genetic marker was also not associated with MM risk in our study. We observed similar genotype distribution in all subject groups, which is consistent with previously published results. ${ }^{28}$

An important finding is that when rs1057147 genotype was taken into account when determining the serum SMRP cutoff value, the diagnostic predictive value was improved both in our present and in the previously published study. ${ }^{28}$ Cutoff values with the highest sum of specificity and sensitivity differed in respective genotype groups. Similar results were reported for SNPs in the $5^{\prime}$ UTR of the MSLN gene that may affect binding of transcription factors: polymorphic alleles were associated with increased SMRP levels only in subjects without $\mathrm{MM}$ and accounting for the genotype improved the diagnostic performance of SMRP. 25,26 These results suggest that cutoff values should be redefined taking into account the MSLN genetic variability to facilitate the interpretation of SMRP measurements. ${ }^{26}$ For example, a lower cutoff used in subjects with the wild-type genotype would decrease false negative results.

We also investigated serum SMRP levels and MSLN rs1057147 as a prognostic biomarker in MM. In our study, serum SMRP alone was not significantly associated with the survival of MM patients, even though patients with SMRP above $1.5 \mathrm{nmol} / \mathrm{l}$ had somewhat shorter OS. Discrepant results were observed in previous studies where very different cutoff values were used. Still, in a meta-analysis, increased SMRP was associated with shorter overall survival, but more comprehensive studies are still needed..$^{18}$ However, several studies suggested that a better predictor than baseline serum SMRP would be the change in serum SMRP determined by lon- gitudinal follow-up of MM patients. ${ }^{22,23,34,35}$ Serum SMRP could thus be used for monitoring disease progression allowing for early reintroduction of chemotherapy. ${ }^{36}$ In Slovenian MM patients, SMRP levels were also increased at disease progression. ${ }^{21}$

On the other hand, MSLN rs1057147 was an independent predictor of OS in MM patients in our study. Carriers of at least one polymorphic allele had significantly shorter OS and tended to have shorter PFS. To the best of our knowledge, this is the first report showing that MSLN genetic variability can influence the survival of MM patients and further studies are needed to explain the influence of this SNP on survival. As the polymorphic MSLN rs1057147 allele was associated with higher serum SMRP levels, this is in agreement with the suggested prognostic role of serum SMRP. The combination of both serum SMRP levels and MSLN genetic variability was the best predictor of OS in our study and could therefore serve as a better composite prognostic biomarker in MM.

One of the shortcomings of the present study was that we did not include SNPs in MSLN 5' UTR that could further contribute to the variability in SMRP levels. Apart from that, serum samples were not available for all MM patients at diagnosis, although we do not believe that any systematic bias was involved with this reduction. Additionally, some studies have discussed that other confounding factors, such as age, body mass index and glomerular filtration rate, may affect SMRP levels. ${ }^{22,37,38}$ In our study, age, gender and smoking were not associated with serum SMRP levels, but data on body mass index and kidney function were not available. However, not all studies confirmed that these factors influence SMRP levels. ${ }^{22,37,38}$ On the other hand, we included a large number of subjects, evaluated different asbestos-related diseases separately and evaluated both serum SMRP levels and MSLN rs1057147 as potential diagnostic and prognostic biomarkers.

Mesothelin is by far the most studied biomarker in MM; however, other diagnostic and prognostic biomarkers were also reported. Among serum biomarkers, especially fibulin-3 and osteopontin are promising candidates, but novel biomarkers, such as plasma biomarkers, HMGB1, mRNA and microRNA expression, are also emerging. ${ }^{16,39-45}$ Additionally, recent studies suggest that a combination of different biomarkers could improve the predictive capacity of SMRP. ${ }^{41,42,44,46,47}$ Our present results show that genetic variability affecting the expression of these biomarkers should also be taken into account. 
In conclusion, our results confirm that MSLN genetic variability affects serum SMRP levels and could contribute to a better definition of cutoff values for MM diagnosis and screening. We were also the first to show that MSLN genetic variability may influence the survival of MM patients. The combination of genetic data and serum biomarkers could therefore serve as a better diagnostic and prognostic biomarker in asbestos-exposed subjects and MM patients.

\section{Acknowledgements and funding}

This work was financially supported by the Slovenian Research Agency (ARRS Grants No. P10170, L3-8203).

\section{References}

1. Johnen G, Chapman SJ, Cookson WO, Musk AW, Lee YC. Benign asbestos pleural diseases. Curr Opin Pulm Med 2003; 9: 266-71. doi: 10.1097/00063198-200307000-00004

2. IARC monographs on the evaluation of the carcinogenic risk of chemicals to man: asbestos. IARC Monogr Eval Carcinog Risk Chem Man 1977; 14: 1-106. PMID: 863456

3. Robinson BWS, Lake RA. Medical progress - advances in malignant mesothelioma. N Eng/ J Med 2005; 353: 1591-603. doi: 10.1056/NEJMra050152

4. Kovac V, Zwitter M, Zagar T. Improved survival after introduction of chemotherapy for malignant pleural mesothelioma in Slovenia: population-based survey of 444 patients. Radiol Oncol 2012; 46: 136-44. doi: 10.2478/ v10019-012-0032-0

5. Damhuis RA, Schroten C, Burgers JA. Population-based survival for malignant mesothelioma after introduction of novel chemotherapy. Eur Respir 2012; 40: 185-9. doi: 10.1183/09031936.00153611

6. Helland A, Solberg S, Brustugun OT. Incidence and survival of malignant pleural mesothelioma in norway: a population-based study of 1686 cases. J Thorac Oncol 2012; 7: 1858-61. doi: 10.1097/JTO.0b013e318275b346

7. Tomasetti M, Santarelli L. Biomarkers for early detection of malignant mesothelioma: diagnostic and therapeutic application. Cancers 2010; 2: 523-48. doi: $10.3390 /$ cancers 2020523

8. Robinson BW, Creaney J, Lake R, Nowak A, Musk AW, de Klerk N, et al. Mesothelin-family proteins and diagnosis of mesothelioma. Lancet 2003, 362: 1612-6. doi: 10.1016/S0140-6736(03)14794-0

9. Husain AN, Colby TV, Ordóñez NG, Allen TC, Attanoos RL, Beasley MB, et al. Guidelines for Pathologic Diagnosis of Malignant Mesothelioma 2017 Update of the Consensus Statement From the International Mesothelioma Interest Group. Arch Pathol Lab Med 2018; 14: 89-108. doi: 10.5858/ arpa.2017-0124-RA

10. Ordonez NG. Value of mesothelin immunostaining in the diagnosis of mesothelioma. Mod Pathol 2003; 16: 192-7. doi: 10.1097/01. MP.0000056981.16578.C3

11. Creaney J, Olsen NJ, Brims F, Dick IM, Musk AW, de Klerk NH, et al. Serum mesothelin for early detection of asbestos-induced cancer malignant mesothelioma. Cancer Epidemiol Biomarkers Prev 2010; 19: 2238-46. doi: 10.1158/1055-9965.EPI-10-0346

12. Hollevoet K, Reitsma JB, Creaney J, Grigoriu BD, Robinson BW, Scherperee $A$, et al. Serum mesothelin for diagnosing malignant pleural mesothelioma: an individual patient data meta-analysis. J Clin Oncol 2012; 30: 1541-9. doi: $10.1200 / \mathrm{JCO} 2011.39 .6671$
13. Hollevoet K, Van Cleemput J, Thimpont J, De Vuyst P, Bosquee L, Nackaerts $\mathrm{K}$, et al. Serial measurements of mesothelioma serum biomarkers in asbestos-exposed individuals: a prospective longitudinal cohort study. J Thorac Oncol 2011; 6: 889-95. doi: 10.1097/JTO.0b013e31820db377

14. Cui A, Jin XG, Zhai K, Tong ZH, Shi HZ. Diagnostic values of soluble mesothelin-related peptides for malignant pleural mesothelioma: updated metaanalysis. BMJ open 2014; 4: e004145. doi: 10.1136/bmjopen-2013-004145

15. Hassan R, Thomas A, Alewine C, Le DT, Jaffee EM, Pastan I. Mesothelin immunotherapy for cancer: ready for prime time? J Clin Oncol 2016; 34: 4171-9. doi: 10.1200/JCO.2016.68.3672

16. Gillezeau C, van Gerwen M, Ramos J, Liu B, Flores R, Taioli E. Biomarkers for malignant pleural mesothelioma: a meta-analysis. Carcinogenesis 2019. doi: 10.1093/carcin/bgz103

17. Robinson BW, Creaney J, Lake R, Nowak A, Musk AW, de Klerk N, et al. Soluble mesothelin-related protein--a blood test for mesothelioma. Lung Cancer 2005; 49(Suppl 1): S109-11. doi: 10.1016/j.lungcan.2005.03.020

18. Tian L, Zeng R, Wang X, Shen C, Lai Y, Wang M, et al. Prognostic significance of soluble mesothelin in malignant pleural mesothelioma: a meta-analysis. Oncotarget 2017; 8: 46425-35. doi: 10.18632/oncotarget.17436

19. He X, Wang L, Riedel H, Wang K, Yang Y, Dinu CZ, et al. Mesothelin promotes epithelial-to-mesenchymal transition and tumorigenicity of human lung cancer and mesothelioma cells. Mol Cancer 2017; 16: 63. doi: 10.1186/ s12943-017-0633-8

20. Cristaudo A, Bonotti A, Simonini S, Bruno R, Foddis R. Soluble markers for diagnosis of malignant pleural mesothelioma. Biomark Med 2011; 5: 26173. doi: $10.2217 / \mathrm{bmm} .11 .18$

21. Franko A, Dolzan V, Kovac V, Arneric N, Dodic-Fikfak M. Soluble mesothelinrelated peptides levels in patients with malignant mesothelioma. Dis Markers 2012; 32: 123-31. doi: 10.3233/DMA-2011-0866

22. de Fonseka $\mathrm{D}$, Arnold DT, Stadon L, Morley $\mathrm{A}$, Keenan $\mathrm{E}$, Darby $\mathrm{M}$, et al. A prospective study to investigate the role of serial serum mesothelin in monitoring mesothelioma. BMC Cancer 2018; 18: 199. doi: 10.1186/ s12885-018-4113-3

23. Linch M, Gennatas S, Kazikin S, lqbal J, Gunapala R, Priest K, et al. A serum mesothelin level is a prognostic indicator for patients with malignant mesothelioma in routine clinical practice. BMC Cancer 2014; 14: 674. doi: 10.1186/1471-2407-14-674

24. Li ZQ, Verch T, Allard WJ. MESOMARK((R)) in vitro diagnostic test for mesothelioma. Expert Opin Med Diagn 2007; 1: 137-42. doi: 10.1517/17530059.1.1.137

25. Cristaudo A, Foddis R, Bonotti A, Simonini S, Vivaldi A, Guglielmi G, et al. Two novel polymorphisms in $5^{\prime}$ flanking region of the mesothelin gene are associated with soluble mesothelin-related peptide (SMRP) levels. Int J Biol Markers 2011; 26: 117-23. doi: 10.5301/JBM.2011.8332

26. De Santi C, Pucci $P$, Bonotti A, Melaiu O, Cipollini M, Silvestri $R$, et al. Mesothelin promoter variants are associated with increased soluble mesothelin-related peptide levels in asbestos-exposed individuals. Occup Environ Med 2017; 74: 456-63. doi: 10.1136/oemed-2016-104024

27. Cristaudo A, Foddis R, Bonotti A, Simonini S, Vivaldi A, Guglielmi G, et al. Polymorphisms in the putative micro-RNA-binding sites of mesothelin gene are associated with serum levels of mesothelin-related protein. Occup Environ Med 2010; 67: 233-6. doi: 10.1136/oem.2009.049205

28. Garritano S, De Santi C, Silvestri R, Melaiu O, Cipollini M, Barone E, et al. A common polymorphism within MSLN affects miR-611 binding site and soluble mesothelin levels in healthy people. J Thorac Oncol 2014; 9: 1662-8. doi: 10.1097/JTO.0000000000000322

29. Tossavainen A. Asbestos, asbestosis, and cancer: the Helsinki criteria for diagnosis and attribution. Scand J Work Environ Health 1997; 23: 311-6. doi: 10.5271/sjweh.226

30. American Thoracic Sociaty Document. Diagnosis and initial management of nonmalignant diseases related to asbestos. Am J Respir Crit Care Med 2004; 170: 691-715. doi: 10.1164/rccm.200310-1436ST

31. Ferris BG. Epidemiology Standardization Project (American Thoracic Society). Am Rev Respir Dis 1978; 118: 1-120. PMID: 742764 
32. Dodic Fikfak M, Kriebel D, Quinn MM, Eisen EA, Wegman DH. A case control study of lung cancer and exposure to chrysotile and amphibole at a slovenian asbestos-cement plant. Ann Occup Hyg 2007; 51: 261-8. doi: 10.1093/ annhyg/mem003

33. Bruno F, Baratti D, Martinetti A, Morelli D, Sottotetti E, Bonini C, et al. Mesothelin and osteopontin as circulating markers of diffuse malignant peritoneal mesothelioma: A preliminary study. Ejso-Eur J Surg Onc 2018; 44: 792-8. doi: 10.1016/j.ejso.2018.02.010

34. Grigoriu BD, Chahine B, Vachani A, Gey T, Conti M, Sterman DH, et al. Kinetics of soluble mesothelin in patients with malignant pleural mesothelioma during treatment. Am J Respir Crit Care Med 2009; 179: 950-4. doi: 10.1164/rccm.200807-11250C

35. Arnold DT, De Fonseka D, Hamilton FW, Rahman NM, Maskell NA. Prognostication and monitoring of mesothelioma using biomarkers: a systematic review. Br J Cancer 2017; 116: 731-41. doi: 10.1038/bjc.2017.22

36. Fontana V, Vigani A, Pistillo MP, Giannoni U, Rosemberg I, Canessa PA, et al. The correlation of serum mesothelin level with pleural thickness in malignant pleural mesothelioma makes it a valuable tool for monitoring tumor progression. J Thorac Oncol 2019; 14: e92-e4. doi: 10.1016/j. jtho.2018.12.026

37. Park EK, Wilson D, Yates DH. A predictive equation to adjust for clinical variables in soluble mesothelin-related protein (SMRP) levels. Clin Chem Lab Med 2012; 50: 2199-204. doi: 10.1515/cclm-2012-0314

38. Filiberti R, Marroni P, Mencoboni M, Mortara V, Caruso P, Cioe A, et al. Individual predictors of increased serum mesothelin in asbestos-exposed workers. Med Oncol 2013; 30: 422. doi: 10.1007/s12032-012-0422-6

39. Pass HI, Levin SM, Harbut MR, Melamed J, Chiriboga L, Donington J, et al. Fibulin-3 as a blood and effusion biomarker for pleural mesothelioma. $N$ Engl J Med 2012; 367: 1417-27. doi: 10.1056/NEJMoa1115050

40. Kovac V, Dodic-Fikfak M, Arneric N, Dolzan V, Franko A. Fibulin-3 as a biomarker of response to treatment in malignant mesothelioma. Radiol Oncol 2015; 49: 279-85. doi: 10.1515/raon-2015-0019

41. Cristaudo A, Bonotti A, Guglielmi G, Fallahi P, Foddis R. Serum mesothelin and other biomarkers: what have we learned in the last decade? J Thorac Dis 2018; 10: S353-9. doi: 10.21037/jtd.2017.10.132

42. Creaney J, Robinson BWS. Malignant mesothelioma biomarkers: from discovery to use in clinical practice for diagnosis, monitoring, screening, and treatment. Chest 2017; 152: 143-9. doi: 10.1016/j.chest.2016.12.004

43. Kirschner MB, Cheng YY, Badrian B, Kao SC, Creaney J, Edelman JJ, et al. Increased circulating miR-625-3p: a potential biomarker for patients with malignant pleural mesothelioma. J Thorac Oncol 2012; 7: 1184-91. doi: 10.1097/JTO.0b013e3182572e83

44. Cristaudo A, Bonotti A, Simonini S, Vivaldi A, Guglielmi G, Ambrosino N, et al. Combined serum mesothelin and plasma osteopontin measurements in malignant pleural mesothelioma. J Thorac Oncol 2011; 6: 1587-93. doi: 10.1097/JTO.0b013e31821e1c08

45. Hoda MA, Dong Y, Rozsas A, Klikovits T, Laszlo V, Ghanim B, et al. Circulating activin $A$ is a novel prognostic biomarker in malignant pleural mesothelioma - a multi-institutional study. Eur J Cancer 2016; 63: 64-73. doi: 10.1016/j. ejca.2016.04.018

46. Foddis R, Bonotti A, Landi S, Fallahi P, Guglielmi G, Cristaudo A. Biomarkers in the prevention and follow-up of workers exposed to asbestos. J Thorac Dis 2018; 10: S360-8. doi: 10.21037/jtd.2017.12.17

47. Santarelli L, Staffolani S, Strafella E, Nocchi L, Manzella N, Grossi P, et al. Combined circulating epigenetic markers to improve mesothelin performance in the diagnosis of malignant mesothelioma. Lung Cancer 2015; 90 457-64. doi: 10.1016/j.lungcan.2015.09.021 